\title{
Shaping nanoparticle diffusion through biological barriers to drug delivery
}

Benjamin J. Lee ${ }^{1}$, Yahya Cheema ${ }^{1}$, Shahed Bader ${ }^{1}$, Gregg A. Duncan ${ }^{1,2^{*}}$

${ }^{1}$ Fischell Department of Bioengineering, University of Maryland, College Park, MD 20742

${ }^{2}$ Biophysics Program, University of Maryland, College Park, MD 20742

*Correspondence to: Gregg Duncan; email: gaduncan@umd.edu

\begin{abstract}
Nanoparticle drug delivery systems encounter many biological barriers, such as the extracellular matrix and mucus gels, that they must bypass to gain access to target cells. A design parameter that has recently gained attention is nanoparticle shape, as it has been shown elongated rod-shaped nanoparticles achieve higher diffusion rates through biological gels. However, the optimal dimensions of rod-shaped nanoparticles to enhance this effect has yet to be established. To systematically approach this, rod-shaped nanoparticles were synthesized by mechanically stretching $100 \mathrm{~nm}, 200 \mathrm{~nm}$, and $500 \mathrm{~nm}$ spherical nanoparticles. Transmission electron microscopy confirmed this procedure yields a significant fraction of elongated rods and remaining spheres could be removed by centrifugation. Fluorescent microscopy and multiple particle tracking analysis was then used to characterize rod-shaped and spherical nanoparticle diffusion in MaxGel®, a model extracellular matrix hydrogel. When dispersed in MaxGel, we found rodshaped nanoparticles exhibited the greatest enhancement in diffusion rate when their length far exceeds the average hydrogel network size. These results further establish the importance of shape as a design criterion to improve nanoparticle-based drug delivery systems.
\end{abstract}

\section{Introduction}

Nanomedicine provides a promising alternative to traditional therapies for efficient drug delivery to selectively target tissues and as contrast agents for diagnostic imaging applications. ${ }^{1}$ Furthermore, nanomedicine-based pharmaceuticals have demonstrated reduced immunogenic and off-target side effects common with traditional therapies. ${ }^{1,} 2$ For pulmonary drug delivery applications, the inhaled administration route has been a target of interest to deliver nanotherapeutics given the high surface area and extensive vascularization of the lung. ${ }^{4-6}$ Despite these benefits, there are various obstacles to be overcome, which if not accounted for can hinder the clinical performance of nanomedicine. ${ }^{2}$ In order to achieve the desired therapeutic outcomes, nanomedicine must efficiently diffuse through and avoid adhesion to biological gels, such as the extracellular matrix (ECM) and mucus, in order to reach target cells within the tissue of interest. For example, ECM possesses a mesh-like structure through the cross-linking and entanglement of collagen fibers and other proteoglycans which can inhibit efficient transport and reduce retention of nanoparticle drug delivery systems. ${ }^{3-5}$ In diseased states such as cancer, the ECM becomes more concentrated making this microenvironment less easily penetrated and presenting a greater barrier to drug delivery. ${ }^{6}$ Likewise, the biodistribution and efficacy of inhaled drug and gene nanocarriers can be significantly limited by mucus secreted on the airway epithelial surface depending on nanoparticle size and surface chemistry. ${ }^{7,8}$ In addition, mucus observed in diseases such as cystic fibrosis and asthma is significantly more concentrated and densely cross-linked, which may further inhibit efficient pulmonary nanoparticle drug delivery. ${ }^{9,} 10$ Taken together, extracellular barriers within target tissues are likely to influence the effectiveness of nanoparticle-based therapies.

Many pathogenic viruses and bacteria have complex, anisotropic shapes which enhance their ability to survive and replicate in their host. ${ }^{11,} 12$ These observations have inspired research exploring shape as a design parameter for drug delivery systems. ${ }^{13}, 14$ Prior work has shown rodshaped nanoparticles can more readily penetrate through biological barriers to delivery. For 
example, it has been shown rod-shaped tobacco mosaic viral filaments achieves more rapid diffusion through $3 \mathrm{D}$ tumor spheroids. ${ }^{15}$ In addition, previous studies have shown that mesoporous silica nanorods exhibit higher diffusion in gastrointestinal (GI) mucus and longer retention in the GI tracts of mice than its spherical counterparts when orally administered. ${ }^{16,17}$ Using molecular simulations, they found that nanorods better penetrate GI mucus due to non-uniform rotational motion and directional transport under shear flow. ${ }^{16}$ For targeted drug delivery applications, it has been demonstrated ligand-functionalized, rod-shaped nanoparticles possess highly specific adhesion to lung and brain endothelium in an in vitro microfluidic model mimicking the vasculature and in an in vivo model where mice were injected with intercellular adhesion molecule monoclonal antibody (ICAM-mAb)-coated nanorods. ${ }^{18,19}$

While these important findings support the use of rod-shaped nanomaterials for biomedical applications, prior studies have yet to systemically characterize rod-shaped nanoparticle behavior in biological barriers to drug delivery, such as the ECM. Herein, we report measurements of nanorod and nanosphere of varying size to find the optimal dimensions for nanoparticle penetration through a model ECM hydrogel. Elongated rod-shaped nanoparticles were prepared using a previously established mechanical stretching technique. ${ }^{20}$ The motions of individual nanoparticles were directly measured using fluorescent microscopy and multiple particle tracking analysis. We show here that nanorod dimensions must be tuned with respect to ECM architecture where increasing nanorod length beyond the ECM mesh pore size leads to marked enhancements in diffusion rate. The diffusion of nanorods with lengths less than or in range of the ECM mesh spacing was left unchanged or reduced as compared to their spherical counterparts. These data provide new insights on the shape dependence of nanoparticle dynamics in biological gels and design considerations in creating nanoparticle drug delivery systems.

\section{Methods}

Mechanical stretching of nanospheres (NS) into nanorods (NR). The protocol described here was adapted from a previously reported method. ${ }^{20}$ To create a film with embedded NS, $20 \mathrm{~mL}$ of 5\% $\mathrm{w} / \mathrm{v}$ solution of polyvinyl alcohol (PVA) was mixed using a magnetic stir bar and heated at $130^{\circ} \mathrm{C}$ for at least one hour until fully dissolved. The solution was passed through a fine mesh grid to remove any undissolved lumps of PVA. Next, $100 \mathrm{~nm}, 200 \mathrm{~nm}$, or $500 \mathrm{~nm}$ fluorescent carboxylcoated polystyrene NS (ThermoFisher) were mixed into the PVA solution at concentration ranging from $0.01-0.1 \% \mathrm{w} / \mathrm{v}$. To plasticize the film, $320 \mu \mathrm{L}$ of glycerol was also added to the PVA/NS containing solution. The solution was then poured into a $12 \times 12 \mathrm{~cm}$ mold and left to dry overnight uncovered. Small bubbles present in the film were removed using tweezers. Once the film fully dried, two $9 \times 5 \mathrm{~cm}$ films were cut from the film and submerged in toluene overnight to liquefy the nanoparticles. Films were taken out, and a $5 \times 5 \mathrm{~cm}$ grid was drawn on the center of the film using a marker to visually determine the uniformity of the stretching. The films were loaded on an Arduino-controlled mechanical stretcher by adhering and clamping the film on the platform using tape and Gorilla Glue. The film was then stretched to up to 3 times the size of the marked grid and left to dry overnight. The film was cut along the grid marks and any non-uniformly stretched portions were discarded. The remaining film sections were submerged in isopropanol to remove any residual toluene. After 24 hours, the film sections were removed and then put into a $30 \%$ isopropanol solution, which was then mixed and heated at $130{ }^{\circ} \mathrm{C}$ for an hour until the film had fully dissolved. The dissolved films were centrifuged for 30 minutes at $26,000 \mathrm{xg}$, and the supernatant was discarded. The pellet was re-suspended in $30 \%$ isopropanol at $130{ }^{\circ} \mathrm{C}$ and after 1 hour, centrifuged at $26,000 \mathrm{xg}$. This process of PVA film dissolution and washing was repeated three times. To separate the rods from the spheres, solutions of stretched NS and NR were put into 
$1.5 \mathrm{~mL}$ centrifuged tubes and spun at 12,000xg for $100 \mathrm{~nm}$ and $200 \mathrm{~nm}$ NS and 10,000xg for 500 $\mathrm{nm}$ NS for 10 minutes. The pellet that accumulated on the side, theoretically containing NR, was disrupted using a spatula and the supernatant was decanted into a separate centrifuge tube. ${ }^{21}$ The remaining pellet containing NS at the bottom of the tube was resuspended in $500 \mu \mathrm{LupH}_{2} \mathrm{O}$.

Transmission Electron Microscopy (TEM). Stretched nanoparticles were diluted 100x in ultrapure $\mathrm{H}_{2} \mathrm{O}$ and $25 \mu \mathrm{L}$ was placed on a square of Parafilm. A PELCO ${ }^{\circledR}$ copper mesh grid $(400$ mesh $\mathrm{Cu}$, Ted Pella) was inverted and placed on this droplet for 30 seconds. After, the grid was washed 4 times for 30 seconds by placing on a $25 \mu \mathrm{L}$ droplet of ultrapure $\mathrm{H}_{2} 0$. After drying, the grid was imaged using transmission electron microscopy (JEM-2100, $200 \mathrm{kV}$, JEOL Ltd).

Fluorescent video microscopy and multiple particle tracking (MPT) analysis. Custom microscopy chambers were prepared as previously described. ${ }^{22}$ Microscopy chambers were either filled with $\mathrm{H}_{2} \mathrm{O}$ for control experiments or $\sim 1 \mathrm{mg} / \mathrm{mL}$ MaxGel (Sigma Aldrich). Slides containing MaxGel were incubated at $37^{\circ} \mathrm{C}$ for 30 minutes to form a gel. After incubation, $1 \mu \mathrm{L}$ of $\sim 1000 \mathrm{x}$ diluted NS or NR was added to $24 \mu \mathrm{L}$ of sample. The slides were covered with a plastic cover slide and left for 30 minutes to allow the nanoparticles to equilibrate in the solution. Slides containing MaxGel were left in the incubator until ready for MPT analysis. Fluorescence video microscopy and MPT analysis was used to determine the translational diffusion of the nanoparticles in aqueous and hydrogel media. MPT experiments were performed using a Zeiss 800 LSM microscope and videos were acquired at $33.33 \mathrm{~Hz}$ frame rate for 10 seconds. Videos were analyzed using TrackPy (github.com/soft-matter/trackpy), a Python-based particle tracking code that detects Gaussian blob-like features in a video. Particle trajectories are then used to calculate time-averaged mean square displacement (MSD) as a function of lag time, $\tau$ and from this, the average translational diffusion coefficient $(D)$ of the nanoparticles can be determined. For a nanoparticle in a Newtonian fluid, $D$ for NS can be calculated from the Stokes-Einstein equation,

$$
D_{\mathrm{NS}}=k_{\mathrm{B}} T / 6 \pi \mu a(1)
$$

where $k_{\mathrm{B}}$ is Boltzmann's constant, $T$ is the temperature, $\mu$ is the viscosity of the solution, and $a$ is the radius of the nanoparticle. When calculating the theoretical rate of diffusion for rodshaped nanoparticles in a purely viscous fluid, a modified version of the Stokes-Einstein Equation must be used to account for anisotropic hydrodynamic effects. Specifically, the diffusion coefficient for NR $\left(D_{N R}\right)$ must account for motion parallel and perpendicular with respect to the long axis and can be calculated as,

$$
D_{\mathrm{NR}}=\left(D_{\|}+2 D_{\perp}\right) / 3
$$

where $D_{\|}=\left(k_{\mathrm{B}} T / 2 \pi \mu\right) \times \ln [L / d], D_{\perp}=\left(k_{\mathrm{B}} T / 4 \pi \mu\right) \times \ln [L / d], L$ is the length of the long axis of the nanorod, and $d$ is the cross-sectional diameter of the shorter axis. ${ }^{23}$ Additional analyses were performed to characterize the viscoelastic properties MaxGel through MPT. The logarithmic slope of MSD $(\alpha)$ can be calculated as $\alpha=\log _{10}(\langle\operatorname{MSD}(\tau)\rangle) / \log _{10}(\tau)$. For a gel network, sub-diffusive nanoparticle movement should be observed where $0<\alpha<1 .{ }^{24}$ Using the generalized StokesEinstein relation, measured MSD values were used to compute viscoelastic properties. The Laplace transform of $\langle\operatorname{MSD}(\tau)\rangle,\langle\operatorname{MSD}(\mathrm{s})\rangle$, is related to viscoelastic spectrum $\widetilde{G}(s)$ using the equation $\left.\widetilde{G}(s)=2 k_{\mathrm{B}} T /[\pi a s \mathrm{MSD}(s)\rangle\right]$, where $s$ is the complex Laplace frequency. The complex modulus can be calculated as $G^{*}(\omega)=G^{\prime}(\omega)+G^{\prime \prime}(i \omega)$, with $i \omega$ being substituted for $s$, where $i$ is a complex number and $\omega$ is frequency. ${ }^{24}$ Hydrogel network pore size, $\xi$, is estimated based on $G$ ' using the equation, $\xi \approx\left(k_{\mathrm{B}} T / \mathrm{G}^{\prime}\right)^{1 / 3} \cdot{ }^{24}$ 


\section{Results \& Discussion}

Mechanical Stretching of Nanoparticles into Ellipsoidal Rod-Shapes. In this work, rod-shaped ellipsoidal nanoparticles were fabricated by unidirectional mechanical stretching of spherical nanoparticles. Films containing fluorescent, carboxyl-coated polystyrene NS of diameters $100 \mathrm{~nm}$, $200 \mathrm{~nm}$, and $500 \mathrm{~nm}$ were stretched by $\sim 3 \mathrm{X}$ their original length. TEM was used to determine the overall uniformity and shape of the nanoparticles after stretching (Fig. 1). TEM micrographs show that the procedure yielded a significant number of rod-shaped ellipsoidal particles, termed here as "nanorods" (NR). The length and width of NR were determined using ImageJ (Table 1).
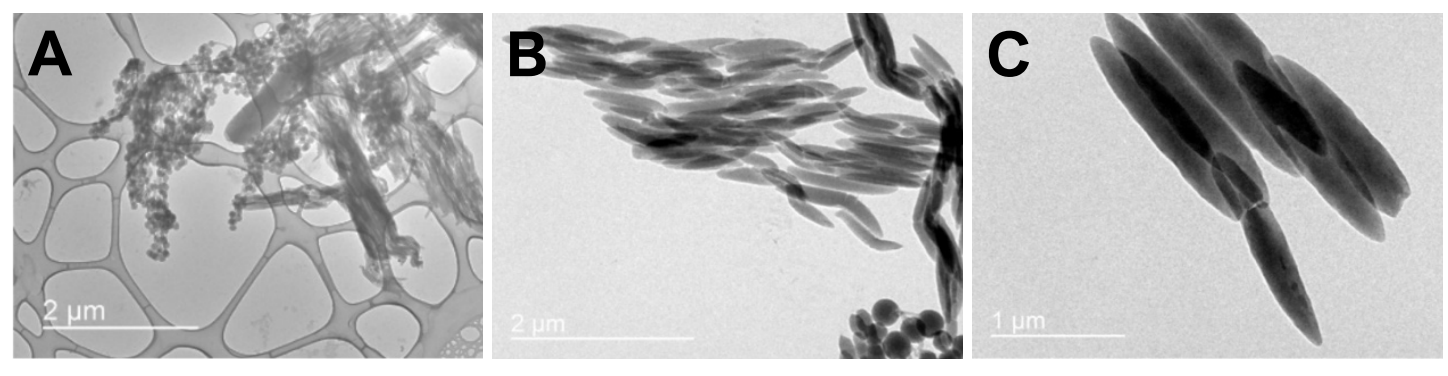

Figure 1. Ellipsoidal nanorod production. Transmission electron microscopy images of (A) $100 \mathrm{~nm}$, (B) $200 \mathrm{~nm}$, and (C) $500 \mathrm{~nm}$ polystyrene nanospheres after mechanical stretching into ellipsoidal nanorods.

Table 1. Average length $(L)$ and width $(d)$ of $100 \mathrm{~nm}, 200 \mathrm{~nm}$, and $500 \mathrm{~nm}$ nanoparticles after stretching The number of nanoparticles (n) measured and standard deviation in $\mathrm{nm}$ are also indicated.

\begin{tabular}{|l|c|c|c|}
\hline \multirow{2}{*}{} & \multicolumn{3}{|c|}{ Original Nanoparticle Diameter } \\
\cline { 2 - 4 } & $\mathbf{1 0 0} \mathbf{~ n m}(\mathbf{n}=\mathbf{1 0 9})$ & $\mathbf{2 0 0} \mathbf{~} \mathbf{n m}(\mathbf{n}=\mathbf{1 0 5})$ & $\mathbf{5 0 0} \mathbf{~} \mathbf{m}(\mathbf{n}=\mathbf{9 9})$ \\
\hline $\boldsymbol{d} \mathbf{( n m})$ & $302 \pm 18.4$ & $705 \pm 132$ & $1770 \pm 208$ \\
\hline
\end{tabular}

Centrifugal separation of nanorods and nanospheres. Based on TEM micrographs (Fig. 1), it was apparent a significant fraction of spherical nanoparticles remained after stretching. In order to generate more purified NR stocks, we adapted a procedure previously shown to separate gold NS and NR by a simple centrifugation step. ${ }^{21}$ Specifically, stretched samples containing both NR and NS were centrifuged to create shape-dependent pellets where NS accumulate on the bottom and NR accumulate on the sides of the centrifuge tube (Fig. 2A) ${ }^{21}$ After the pellets were formed, NR on the side were carefully removed from the wall and put into a separate tube. NS within the bottom pellet were re-dispersed in water in the same tube. It was also apparent the amount of unstretched particles remaining was most significant after stretching of $100 \mathrm{~nm}$ NS (Fig 1A). As such, we subjected these $100 \mathrm{~nm}$-based NR samples to MPT analysis to assess the uniformity of the recovered NS fraction based on their diffusion in water as compared to stock NS. MSD of the NS recovered by centrifugation and stock NS were calculated and diffusion coefficients were determined based on linear regression (Fig. 2B). We find the MSD and diffusion coefficient are very similar for stock and recovered NS with a predicted diameter of 115 and $114 \mathrm{~nm}$, respectively (Eq. 1). We next compared the MSD and diffusion rate of NR recovered by centrifugation to stock NS (Fig. 2C). We find, as expected, NR diffuse more slowly than NS in water based on measured MSD. Assuming an aspect ratio $(L / d)$ of 5, we fit for an effective diffusion coefficient for the NR using Eq. 2 and find the predicted dimensions of $N R(L=355 \mathrm{~nm} ; \mathrm{d}=71 \mathrm{~nm})$ are similar in magnitude to our TEM measurements (Table 1). These results support centrifugal separation of 
stretched NS sample yields a higher purity of NR than what was observed through TEM. As such, we used centrifugal purification in our subsequent studies on NR diffusion in ECM hydrogels.
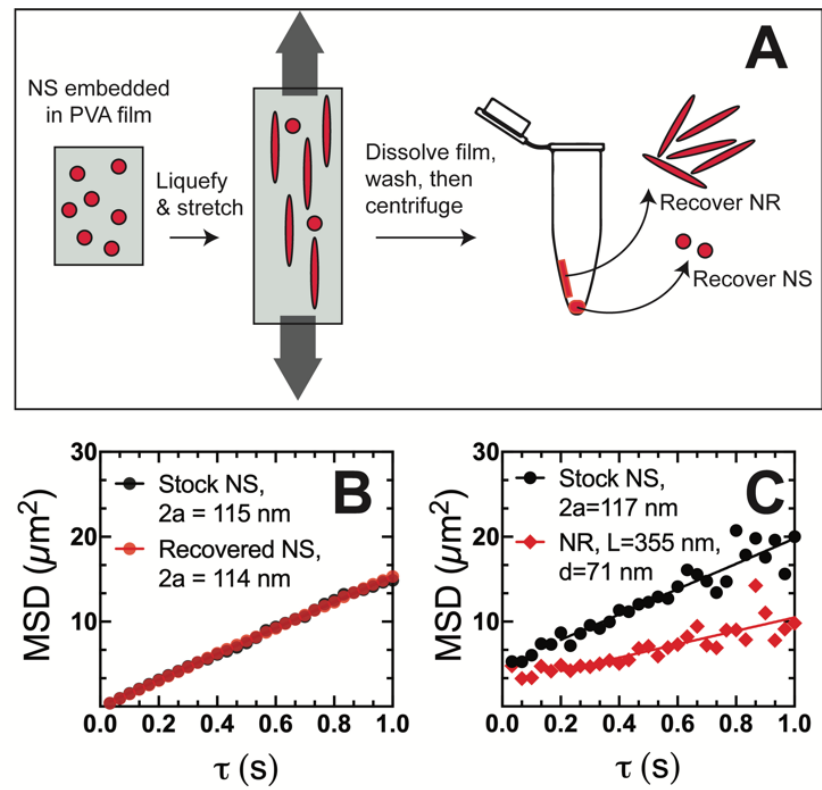

Figure 2. Diffusion of stretched nanorods (NR) and unstretched nanospheres (NS) isolated by centrifugation compared to as-prepared stock NS. (A) Illustration of the stretching procedure and isolation of nanospheres (NS) and nanorods (NR) by centrifugation. (B, C) Multiple particle tracking was conducted for NS and NR in water to determine their respective sizes based on measured mean squared displacement (MSD) and diffusion rate $(D)$. (B) MSD versus time lag $(\tau)$ of unstretched stock NS and recovered spherical nanoparticles (NS) separated by centrifugation in water. $(\mathbf{C})$ MSD versus time lag $(\tau)$ of unstretched stock NS and recovered NR separated by centrifugation. The diameter $(2 a)$ of NS was calculated from measured $D_{\mathrm{NS}}$ using Equation 1. The length $(L)$ and diameter $(d)$ of NR were determined based on measured $D_{\mathrm{NR}}$ using Equation 2 assuming an aspect ratio $(L / d)$ of 5 .

Microrheology and network structure of ECM hydrogels (MaxGel). To gain insights on the behavior of NR and NS in an ECM-like environment, we chose to use MaxGel as a model as it is composed of fibroblast-derived, human ECM proteins such as collagen, laminin, and other glycosaminoglycans. MaxGel has been used in prior studies as a tissue culture scaffold to recapitulate the ECM microenvironment. ${ }^{25,26}$ In order to characterize the microscale mechanical properties of MaxGel, we employed MPT microrheology using $100 \mathrm{~nm}$ nanoparticles coated with polyethylene glycol (PEG), prepared as described in previous work ${ }^{22}$ (Fig. 3). We find based on measured MSD that these nanoparticles exhibited sub-diffusive motion based on the logarithmic slope of MSD versus time, $\alpha=\log [\mathrm{MSD}] / \log [\tau]<1$ (Fig. 3A). Sub-diffusive motion is expected for nanoparticles embedded within a 3D polymer network. Next, we quantified the microrheological properties of MaxGel using the generalized Stokes-Einstein equation to find microscale elastic (G') and viscous (G') moduli (Fig. 3B,C). Both G' and G" increased as a function of frequency and $G^{\prime}$ exceeds $G$ " at all measured frequencies which is characteristic of hydrogel networks with viscoelastic properties. However, we should note our measurements are limited to relatively low frequencies $(\omega \leq 1 \mathrm{~Hz})$ using MPT microrheology. ${ }^{24} \mathrm{We}$ estimated network size $(\xi)$ within MaxGel based on measured $G^{\prime}$ at $\omega=1 \mathrm{~Hz}$ for individual nanoparticles (Fig. 3D) which varied over a range between $\sim 200-600 \mathrm{~nm}\left(\xi_{\text {avg }}=415 \mathrm{~nm}\right)$. This heterogeneity in measured $\xi$ is expected for a multi-component ECM hydrogel. These measurements are important 
as they provide a basis for comparison between the NR and NS of varying size to MaxGel network dimensions.
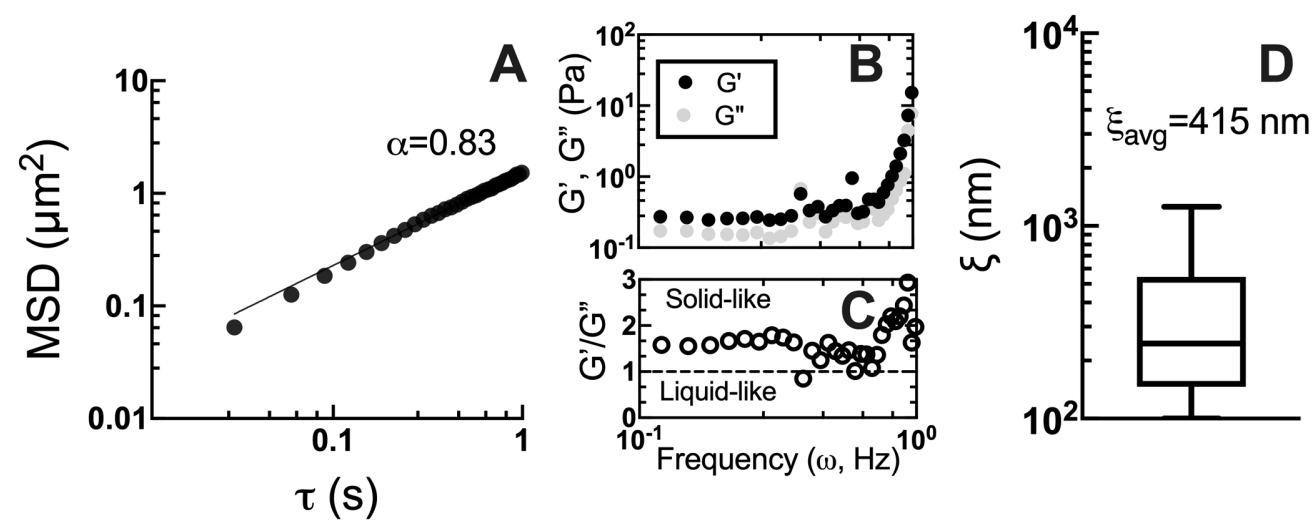

Figure 3. Microscale viscoelastic properties of ECM hydrogels (MaxGel). (A) Mean squared displacement (MSD) versus time with log-log scaling for PEGylated $100 \mathrm{~nm}$ NS in MaxGel. The logarithmic slope of the MSD $(\alpha=\log [\mathrm{MSD}] / \log [\tau])$ is also indicated where values below 1 are indicative of sub-diffusive motion. (B) Elastic (G') and viscous moduli (G') and (C) $G^{\prime} / G^{\prime}$ ' as a function of frequency $(\omega)$ based on microrheological analysis of measured MSD. Values of G'/G" greater than 1 are indicative of elastic solid-like behavior and values less than 1 are indicative of viscous liquid like behavior. (D) Estimated pore size $(\xi)$ based on analysis of $G^{\prime}$ at $\omega=1 \mathrm{~Hz}$ calculated as $\xi \approx\left(k_{\mathrm{B}} T / G^{\prime}\right)^{1 / 3}$.

Shape-dependent nanoparticle diffusion in ECM hydrogels (MaxGel). Finally, the diffusion of NR generated by mechanical stretching and as-prepared stock NS were measured in MaxGel using MPT. We note NS and NR were added to solidified MaxGel following 30 minutes of incubation at $37^{\circ} \mathrm{C}$. The NS and NR were dispersed within the gel by mechanical mixing and were allowed to equilibrate for an additional 30 minutes at $37^{\circ} \mathrm{C}$ prior to imaging. In addition, separate micro-well chambers containing MaxGel were prepared for each NS and NR tested as they fluoresce at the same wavelength. As we observed non-uniform behavior of NS and NR within MaxGel, we compared the overall distribution of MSD at $\tau=1 \mathrm{~s}\left(\mathrm{MSD}_{1 \mathrm{~s}}\right)$ for NS and NR (Fig. 4). Given their comparable surface areas, we compared the diffusion of stock NS to NR prepared using NS of the

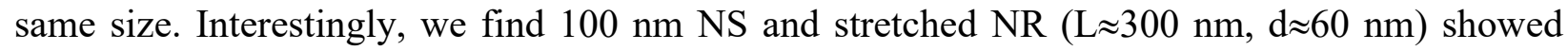
comparable diffusion rates (Fig. 4A). Further, $200 \mathrm{~nm}$ NS-based NR (L $\approx 700 \mathrm{~nm}, \mathrm{~d} \approx 125 \mathrm{~nm}$ ) were more restricted within MaxGel as compared to the original $200 \mathrm{~nm}$ NS (Fig. 4B). Conversely, we observed an enhancement in diffusion rate for the largest $500 \mathrm{~nm}$ NS-based NR $(\mathrm{L} \approx 1.8 \mu \mathrm{m}, \mathrm{d} \approx 285$ $\mathrm{nm}$ ) as compared to $500 \mathrm{~nm}$ NS (Fig. 4C). For the NS, we observed the expected trend that smaller NS generally diffused faster than larger NS. However, when comparing NR, the $\sim 1.8 \mu \mathrm{m}$ long NR diffused the most rapidly overall and $\sim 700 \mathrm{~nm}$ long NR exhibited the slowest diffusion rate.

These results can be interpreted based on prior experimental and computational studies demonstrating constrained motion perpendicular to the long axis can lead to enhancements in translational diffusion through a polymer mesh network. ${ }^{24,27,28}$ For $\sim 300 \mathrm{~nm} \mathrm{NR}$, their motion is comparable to $100 \mathrm{~nm}$ NS given their overall dimensions are smaller than the average network spacing in MaxGel $\left(\mathrm{d}<\mathrm{L}<\xi_{\text {avg }}\right)$. For $\sim 700 \mathrm{~nm} \mathrm{NR}$, although in the size range $\left(\mathrm{d}<\xi_{\text {avg }}<\mathrm{L}\right)$ where we would predict faster diffusion due to reduced frictional drag parallel to the long axis, we do not observe enhanced diffusion which may be due in part to the natural heterogeneity of the ECM network and wide range of pore size which may interfere with these effects. The larger $\sim 1.8 \mu \mathrm{m}$ NR are in the predicted regime where NR diffusion is increased due to reduced hydrodynamic drag 
parallel to the long axis based on the comparative size of NR and $\xi\left(\mathrm{d}<\xi_{\text {avg }}<<\mathrm{L}\right)$. These observations would suggest that NR length should far exceed network pore sizes within the ECM to achieve enhanced penetration through the ECM. However, additional work is required to determine how disease-associated changes to ECM (e.g. increased concentration and/or crosslinking) would impact NR penetration and if these results are generalizable to other biological barriers (e.g. mucus). It will also be of interest in future work to determine if these findings may apply to flexible $\mathrm{NR}^{29,30}$ as compared to the rigid NR studied here and how surface chemistry of NR influences diffusion behavior within ECM and other biological barriers to drug delivery. ${ }^{31,32}$

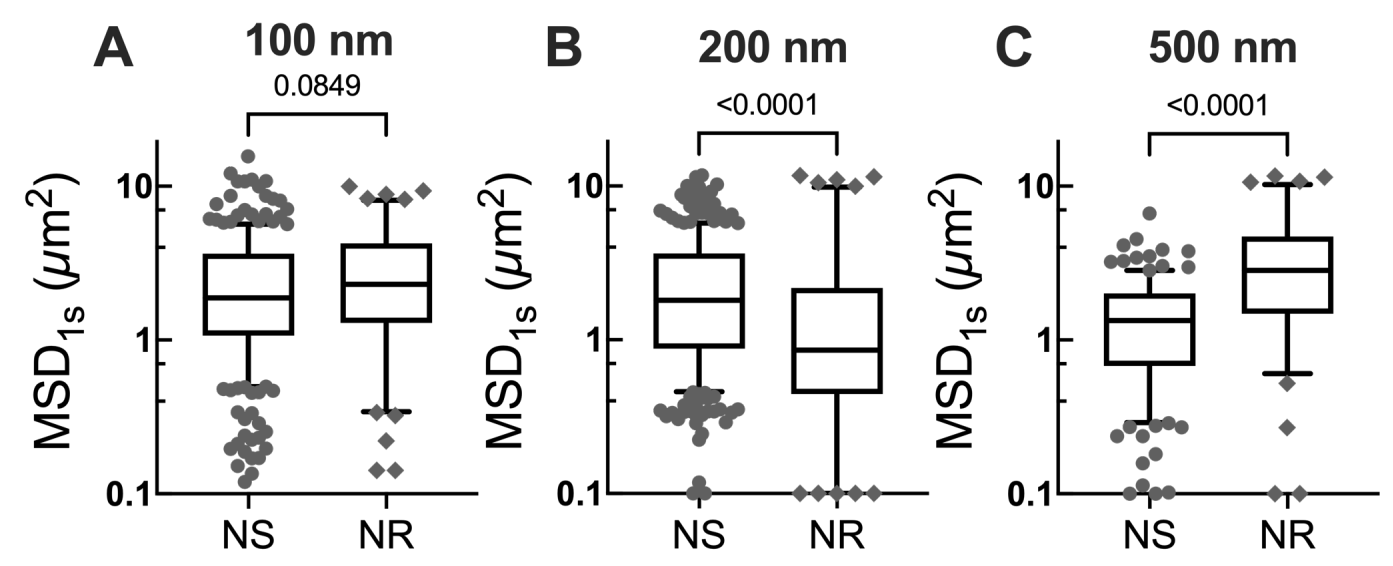

Figure 4. Nanosphere and nanorod diffusion within ECM hydrogels (MaxGel). Measured mean squared displacement at $\tau=1 \mathrm{~s}\left(\mathrm{MSD}_{1 \mathrm{~s}}\right)$ of nanospheres (NS) and nanorods (NR) within MaxGel. Data are shown for NS and NR with original diameters of (A) $100 \mathrm{~nm},(\mathbf{B}) 200 \mathrm{~nm}$, and (C) $500 \mathrm{~nm}$ in MaxGel. Experiments were repeated at least in triplicate with 10-20 videos analyzed and 100-300 individual particles tracked per condition. Mann-Whitney U test was used to compare NS and NR MSD $\mathrm{s}_{1 \mathrm{~s}}$ where $p$ value less than 0.05 was considered significant.

\section{Conclusion}

This work provides new insights into how shape influences the dynamic behavior of nanoparticles within a model ECM hydrogel. While our stretching procedure yielded a mixture of rods and spheres, we demonstrated rod-shaped particles could be purified using centrifugation. Clear differences were observed in the diffusion of rod-shaped and spherical nanoparticles within MaxGel which were dependent on how the length of rod-shaped nanoparticles compared to the ECM mesh spacing. Our results underscore the significance of the ECM as a barrier to nanoparticle drug delivery systems and the potential benefit of controlling shape to improve their distribution within target tissues.

\section{Conflicts of interest}

The authors declare no conflict of interest.

\section{Declaration of competing interest}

The authors declare that they have no known competing financial interests or personal relationships that could have appeared to influence the work reported in this paper. 


\section{Acknowledgements}

This work was supported by the University of Maryland, the Burroughs Wellcome Fund Career Award at the Scientific Interface, the American Lung Association Innovation Award, and the NSF CAREER Award 2047794. We also acknowledge the support of the Maryland NanoCenter and its AIMLab for access to and assistance with TEM imaging.

\section{References}

1. Riehemann, K.; Schneider, S. W.; Luger, T. A.; Godin, B.; Ferrari, M.; Fuchs, H., Nanomedicine_challenge and perspectives. Angewandte Chemie International Edition 2009, 48 (5), 872-897.

2. Barua, S.; Mitragotri, S., Challenges associated with penetration of nanoparticles across cell and tissue barriers: a review of current status and future prospects. Nano today 2014, 9 (2), 223-243.

3. Mohanty, R. P.; Liu, X.; Kim, J. Y.; Peng, X.; Bhandari, S.; Leal, J.; Arasappan, D.; Wylie, D. C.; Dong, T.; Ghosh, D., Identification of peptide coatings that enhance diffusive transport of nanoparticles through the tumor microenvironment. Nanoscale 2019, 11 (38), 17664-17681.

4. Engin, A. B.; Nikitovic, D.; Neagu, M.; Henrich-Noack, P.; Docea, A. O.; Shtilman, M. I.; Golokhvast, K.; Tsatsakis, A. M., Mechanistic understanding of nanoparticles' interactions with extracellular matrix: the cell and immune system. Particle and fibre toxicology 2017, 14 (1), 1-16.

5. Goodman, T. T.; Olive, P. L.; Pun, S. H., Increased nanoparticle penetration in collagenasetreated multicellular spheroids. International journal of nanomedicine 2007, 2 (2), 265.

6. Zhang, Y. R.; Lin, R.; Li, H. J.; He, W. 1.; Du, J. Z.; Wang, J., Strategies to improve tumor penetration of nanomedicines through nanoparticle design. Wiley Interdisciplinary Reviews: Nanomedicine and Nanobiotechnology 2019, 11 (1), e1519.

7. Schneider, C. S.; Xu, Q.; Boylan, N. J.; Chisholm, J.; Tang, B. C.; Schuster, B. S.; Henning, A.; Ensign, L. M.; Lee, E.; Adstamongkonkul, P., Nanoparticles that do not adhere to mucus provide uniform and long-lasting drug delivery to airways following inhalation. Science advances 2017, 3 (4), e1601556.

8. Murgia, X.; Pawelzyk, P.; Schaefer, U. F.; Wagner, C.; Willenbacher, N.; Lehr, C.-M., Size-limited penetration of nanoparticles into porcine respiratory mucus after aerosol deposition. Biomacromolecules 2016, 17 (4), 1536-1542.

9. Duncan, G. A.; Jung, J.; Hanes, J.; Suk, J. S., The Mucus Barrier to Inhaled Gene Therapy. Molecular Therapy 2016, 24 (12), 2043.

10. Song, D.; Cahn, D.; Duncan, G. A., Mucin Biopolymers and Their Barrier Function at Airway Surfaces. Langmuir: the ACS journal of surfaces and colloids 2020, 36 (43), 12773-12783.

11. Yang, D. C.; Blair, K. M.; Salama, N. R., Staying in shape: the impact of cell shape on bacterial survival in diverse environments. Microbiology and Molecular Biology Reviews 2016, 80 (1), 187-203.

12. Li, T.; Li, Z.; Deans, E. E.; Mittler, E.; Liu, M.; Chandran, K.; Ivanovic, T., The shape of pleomorphic virions determines resistance to cell-entry pressure. Nature Microbiology 2021, $6(5), 617-629$.

13. Safari, H.; Kelley, W. J.; Saito, E.; Kaczorowski, N.; Carethers, L.; Shea, L. D.; EniolaAdefeso, O., Neutrophils preferentially phagocytose elongated particles-An opportunity for selective targeting in acute inflammatory diseases. Science advances 2020, 6 (24), eaba1474.

14. Wibroe, P. P.; Anselmo, A. C.; Nilsson, P. H.; Sarode, A.; Gupta, V.; Urbanics, R.; Szebeni, J.; Hunter, A. C.; Mitragotri, S.; Mollnes, T. E., Bypassing adverse injection 
reactions to nanoparticles through shape modification and attachment to erythrocytes. Nature nanotechnology 2017, 12 (6), 589.

15. Lee, K. L.; Hubbard, L. C.; Hern, S.; Yildiz, I.; Gratzl, M.; Steinmetz, N. F., Shape matters: the diffusion rates of TMV rods and CPMV icosahedrons in a spheroid model of extracellular matrix are distinct. Biomaterials science 2013, 1 (6), 581-588.

16. Yu, M.; Wang, J.; Yang, Y.; Zhu, C.; Su, Q.; Guo, S.; Sun, J.; Gan, Y.; Shi, X.; Gao, H., Rotation-facilitated rapid transport of nanorods in mucosal tissues. Nano letters 2016, $16(11)$, 7176-7182.

17. Zhao, Y.; Wang, Y.; Ran, F.; Cui, Y.; Liu, C.; Zhao, Q.; Gao, Y.; Wang, D.; Wang, S., A comparison between sphere and rod nanoparticles regarding their in vivo biological behavior and pharmacokinetics. Scientific reports 2017, 7 (1), 1-11.

18. Kolhar, P.; Anselmo, A. C.; Gupta, V.; Pant, K.; Prabhakarpandian, B.; Ruoslahti, E.; Mitragotri, S., Using shape effects to target antibody-coated nanoparticles to lung and brain endothelium. Proceedings of the National Academy of Sciences 2013, 110 (26), 10753-10758.

19. Barua, S.; Yoo, J.-W.; Kolhar, P.; Wakankar, A.; Gokarn, Y. R.; Mitragotri, S., Particle shape enhances specificity of antibody-displaying nanoparticles. Proceedings of the National Academy of Sciences 2013, 110 (9), 3270-3275.

20. Champion, J. A.; Katare, Y. K.; Mitragotri, S., Making polymeric micro-and nanoparticles of complex shapes. Proceedings of the National Academy of Sciences 2007, 104 (29), 11901 11904.

21. Sharma, V.; Park, K.; Srinivasarao, M., Shape separation of gold nanorods using centrifugation. Proceedings of the National Academy of Sciences 2009, 106 (13), 4981-4985.

22. Hawkins, R. F.; Duncan, G. A., Plate reader microrheology. AIP Advances 2020, 10 (11), 115312.

23. Choi, J.; Cargnello, M.; Murray, C. B.; Clarke, N.; Winey, K. I.; Composto, R. J., Fast nanorod diffusion through entangled polymer melts. ACS Macro Letters 2015, 4 (9), 952-956.

24. Joyner, K.; Yang, S.; Duncan, G. A., Microrheology for biomaterial design. $A P L$ bioengineering 2020, 4 (4), 041508.

25. Asthana, A.; Kisaalita, W. S., Microtissue size and hypoxia in HTS with 3D cultures. Drug discovery today 2012, 17 (15-16), 810-817.

26. Tieng, V.; Stoppini, L.; Villy, S.; Fathi, M.; Dubois-Dauphin, M.; Krause, K.-H., Engineering of midbrain organoids containing long-lived dopaminergic neurons. Stem cells and development 2014, 23 (13), 1535-1547.

27. Chhetri, R. K.; Blackmon, R. L.; Wu, W.-C.; Hill, D. B.; Button, B.; Casbas-Hernandez, P.; Troester, M. A.; Tracy, J. B.; Oldenburg, A. L., Probing biological nanotopology via diffusion of weakly constrained plasmonic nanorods with optical coherence tomography. Proceedings of the National Academy of Sciences 2014, 111 (41), E4289-E4297.

28. Karatrantos, A.; Composto, R. J.; Winey, K. I.; Clarke, N., Nanorod diffusion in polymer nanocomposites by molecular dynamics simulations. Macromolecules 2019, 52 (6), 25132520.

29. Smith, M.; Poling-Skutvik, R.; Slim, A. H.; Willson, R. C.; Conrad, J. C., Dynamics of Flexible Viruses in Polymer Solutions. Macromolecules 2021, 54 (10), 4557-4563.

30. Fakhri, N.; MacKintosh, F. C.; Lounis, B.; Cognet, L.; Pasquali, M., Brownian motion of stiff filaments in a crowded environment. Science 2010, 330 (6012), 1804-1807.

31. Wan, J.; Wang, J.-H.; Liu, T.; Xie, Z.; Yu, X.-F.; Li, W., Surface chemistry but not aspect ratio mediates the biological toxicity of gold nanorods in vitro and in vivo. Scientific reports 2015, 5 (1), 1-16. 
bioRxiv preprint doi: https://doi.org/10.1101/2021.07.21.453209; this version posted July 22, 2021. The copyright holder for this preprint (which

was not certified by peer review) is the author/funder, who has granted bioRxiv a license to display the preprint in perpetuity. It is made available under aCC-BY-NC-ND 4.0 International license.

32. Jin, S.; Ma, X.; Ma, H.; Zheng, K.; Liu, J.; Hou, S.; Meng, J.; Wang, P. C.; Wu, X.; Liang, X.-J., Surface chemistry-mediated penetration and gold nanorod thermotherapy in multicellular tumor spheroids. Nanoscale 2013, 5 (1), 143-146. 